\title{
Diferencias de personalidad entre adictos a sustancias y población general. Estudio con el TCI-R de casos clínicos con controles emparejados
}

\author{
Eduardo José Pedrero Pérez*; Gloria Rojo Mota** \\ * Doctor en Psicología \\ * Profesora de Terapia Ocupacional. Universidad Rey Juan Carlos. \\ Cad 4 San Blas. Instituto de Adicciones. Ayuntamiento de Madrid. \\ Enviar correspondencia a: \\ Eduardo J. Pedrero Pérez. C/ Alcalá, 527. 28027 MADRID. ESPAÑA. Tel.: 609587233. C.E.: ejpedrero@yahoo.es
}

Recibido: Septiembre 2007 Aceptado: Abril de 2008

\section{RESUMEN}

Objetivo: Explorar diferencias en rasgos de personalidad entre sujetos adictos a sustancias en tratamiento y sujetos de población general emparejados en las variables: sexo, edad y nivel de estudios.

Material y método: Se obtuvo por muestreo consecutivo una muestra de sujetos que inician tratamiento por abuso/ dependencia de sustancias en un centro público (CAD 4). Mediante la técnica de "bola de nieve" se obtuvo una muestra de población general. Se consideraron únicamente las parejas (un sujeto de cada muestra) que presentan la misma edad, el mismo sexo y el mismo nivel de estudios. De este modo se obtuvieron 55 parejas. Se utilizó el TCl-R de Cloninger y se compararon los resultados globales, cruzando las diversas variables.

Resultados: Solo aparecieron diferencias significativas y consistentes en dos rasgos: Búsqueda de Novedad, mayor en adictos, y Autodirección, mayor en población general.

Conclusiones: Los datos sugieren que las diferencias observadas no son previas, sino consecutivas al consumo, pareciendo que son los efectos de las sustancias y las condiciones psicosociales asociadas al consumo las que exacerban o impiden el desarrollo de los rasgos. Se relacionan los hallazgos con estudios que vinculan estas variables con funciones neuropsicológicas y se propone la inclusión de esta perspectiva neuropsicológica en la evaluación y tratamiento de los trastornos adictivos.

Palabras clave: personalidad, TCI-R, búsqueda de novedad, autodirección, temperamento, carácter, función ejecutiva, evaluación, tratamiento.

Palabras clave: personalidad, TCl-R, búsqueda de novedad, autodirección, temperamento, carácter, función ejecutiva, evaluación, tratamiento.

\section{ABSTRACT}

Objective: This article explores differences in personality traits between subjects with substance addiction in treatment and subjects from the general population matched in the variables sex, age and educational level.

Material and method: Consecutive sampling was used to obtain a sample of participants undergoing treatment for addictive disorders at a public centre (CAD 4). A sample of the general population was obtained by means of the snowball technique. Only pairs (one participant from each sample) with the same age, sex and educational level were considered. In this way, 55 pairs were obtained. Cloninger's TCl-R was used and the global results were compared, crossing all variables.

Results: Significant and consistent differences were found in just two traits: Novelty-Seeking, greater in addicts, and SelfDirectedness, greater in general population.

Conclusions: Data strongly suggest that observed differences are not previous, but consecutive to the addiction, it being apparent that the effects of the substances and the psychosocial conditions associated with their consumption are what exacerbates or blocks development of the traits. Findings are related to studies linking these variables with neuropsychological functions, and the inclusion of this neuropsychological perspective is strongly proposed for application to the assessment and treatment of addictive disorders.

Key words: personality, TCI-R; novelty-seeking, selfdirectedness, temperament, character, executive function, assessment, treatment. 


\section{INTRODUCCIÓN}

E estudio de la personalidad en sujetos adictos a sustancias se revela, en el momento actual, como una de las claves principales para comprender la relación que media entre el sujeto, la droga y el ambiente en el que se desarrolla la conducta adictiva. La idea no es nueva: hace más de 70 años que un autor español, Valenciano, proponía ya que "de forma general se admite que el factor causal fundamental de las toxicomanías reside en la personalidad del toxicómano y que solamente teniendo en cuenta condiciones generales e individuales, internas y externas, esenciales y accidentales, puede comprenderse el proceso total de la toxicomanía" (Valenciano, 1936; citado en Cervera, Haro, Martínez-Raga, Bolinches, de Vicente y Valderrama, 2001). Tal estudio se ha efectuado a partir de dos premisas: (1) entendiendo que existe una personalidad presente en todos los adictos a drogas, en forma similar y en condiciones equiparables (McLellan, 1979); (2) considerando los rasgos o combinaciones de rasgos que favorecen la conducta adictiva (Esbaugh, 1982).

Mientras la primera perspectiva ha sido descartada, la segunda ha generado un importante caudal de investigación con resultados diversos. Schuckit, Klein, Twitchell y Smith (1994), en un estudio longitudinal, y utilizando diversas pruebas (EPQ, MMPI y otros), no encontraron diferencias entre aquellos que desarrollaron problemas con el consumo de alcohol y aquellos que no los desarrollaron. Tampoco las encontraron Sánchez-Hervás y Berjano (1996) utilizando el 16-PF, en un grupo de adictos a heroína en tratamiento frente a un grupo control equiparado en edad, sexo y nivel de estudios. Utilizando el MBTI, Janowsky, Hong, Morter y Howe (1999) encontraron muy pocas diferencias entre alcohólicos y población normativa (mayor sensitividad y menor intutividad en adictos), pero amplias y generalizadas cuando al trastorno adictivo se sumaba un síndrome depresivo. Marchiori, Loschi, Marconi, Mioni y Pavan (1999) tampoco encontraron diferencias significativas entre alcohólicos y no alcohólicos en diversas variables psicológicas, como apego y locus de control.

Por el contrario, también son abundantes los estudios en los que se encontraron amplias y consistentes diferencias en rasgos y patrones de personalidad entre adictos y no adictos. Gossop y Eysenck (1980) sugirieron que el rasgo de Neuroticismo podría ser la clave principal para distinguir a adictos de sujetos controles. El Modelo de Cinco Factores de la Personalidad ha sido aplicado a muestras de adictos, encontrándose apoyo consistente a la propuesta sobre elevados niveles de Neuroticismo, pero también bajo Tesón y baja Afabilidad en adictos a diversas sustancias, tanto cuando se utilizó el NEO-PI-R (Brooner, Schmidt y Herbst, 2002; Terracciano y Costa, 2004) como cuan- do se aplicó el BFQ (Berrocal, Ortiz, Fierro y Jiménez, 2001; Pedrero, 2002, 2003, 2007b).

El modelo psicobiológico de Cloninger también se ha aplicado al estudio de las diferencias de personalidad entre adictos y no adictos. Este modelo se sustenta en la existencia de cuatro dimensiones de temperamento (Búsqueda de Novedad, Evitación del Daño, Dependencia de Recompensa y Persistencia), que se suponen más vinculadas a factores biológicos (herencia, neurotrasmisión) y tres rasgos caracteriales (Autodirección, Cooperatividad y Autotrascendencia), más vinculados a factores experienciales y de aprendizaje. En una muestra con 117 varones en tratamiento a los que se aplicó la versión inicial del TCl (Barrón, Mateos y Martínez-Villate, 2004) se encontró que los consumidores de heroína obtenían puntuaciones menores que un grupo control en Dependencia de Recompensa, Autodirección y Cooperatividad y sólo diferencias significativas en algunas subescalas de Búsqueda de Novedad y Evitación del Daño. El grupo de adictos a cocaína presentó puntuaciones mayores que el grupo control en Búsqueda de Novedad y menores en Cooperatividad y Autodirección. Un estudio con 316 sujetos adictos a diversas sustancias, en el que se utilizó la última versión revisada del TCl, el TCI-R (Pedrero, 2006a), encontró diferencias significativas con la población normativa en todas las dimensiones del cuestionario, salvo la Dependencia de Recompensa, observándose que los adictos puntuaron más en Búsqueda de Novedad, Evitación del Daño y Autotrascendencia, y menos en Persistencia, Autodirección y Cooperatividad. Similares resultados se encontraron utilizando una versión reducida del TCl-R con 275 sujetos en tratamiento (Pedrero, 2006b).

Uno de los problemas metodológicos comunes a la mayor parte de los estudios es que las puntuaciones de los sujetos adictos se comparan a poblaciones normativas que, en muchos casos, no presentan la variabilidad necesaria. Además, la comparación de medias no suele tener en cuenta la distribución de las variables en relación a otras, que pueden interferir en la interpretación de los datos. Las variables temperamentales no son estables a lo largo del ciclo vital, presentando algunas una distribución en $U$ (p.e., la Evitación del Daño) o declinando con la edad (p.e., la Búsqueda de Novedad). Los diferentes niveles de escolarización y cualificación académica suponen historiales diferenciales que pueden modificar los rasgos caracteriales. Existen constatadas diferencias de género en personalidad, independientemente del modelo desde el que se efectúe la evaluación, lo que obliga a baremar por separado a varones y mujeres.

De cara a la clínica, se revela de especial interés conocer los rasgos de personalidad más vinculados a la adicción, en todos sus estadios: adquisición, establecimiento del hábito, abuso, dependencia y abandono. Por ello, se requieren estudios que afinen en 
la identificación de estas variables eliminando los sesgos y resultados artefactuales que emergen de la ausencia de control de variables intervinientes. El objetivo del presente trabajo es buscar las diferencias en el temperamento y el carácter entre sujetos que se encuentran en tratamiento por trastornos adictivos y sujetos de población general, equiparados en las variables sexo, edad y nivel de estudios.

\section{MÉTODO}

\section{Participantes}

Se obtuvo una muestra de 110 sujetos, 55 de ellos en tratamiento por politoxicomanía y 55 de población general. Todos ellos quedaron emparejados por edad, sexo y nivel académico alcanzado. Ambos grupos tenían una media de edad de 33,9 \pm 8,9 años, en un rango entre los 16 y los 52 (la distribución por edades se muestra en la Figura 1). Cada grupo está compuesto por 35 varones y 20 mujeres. El 18,2\% de cada grupo sólo tiene estudios primarios, el 29,1\% ha cursado estudios secundarios y el $52,7 \%$ ha iniciado, al menos, estudios universitarios. Las drogas que motivan la demanda de tratamiento son la heroína $(\mathrm{N}=6)$, la cocaína ( $\mathrm{N}=19)$, el alcohol $(\mathrm{N}=23)$, el cannabis $(\mathrm{N}=6)$ o las benzodiacepinas ( $\mathrm{N}=1$ ). Todos los participantes firmaron el consentimiento informado para la utilización anónima de sus resultados.

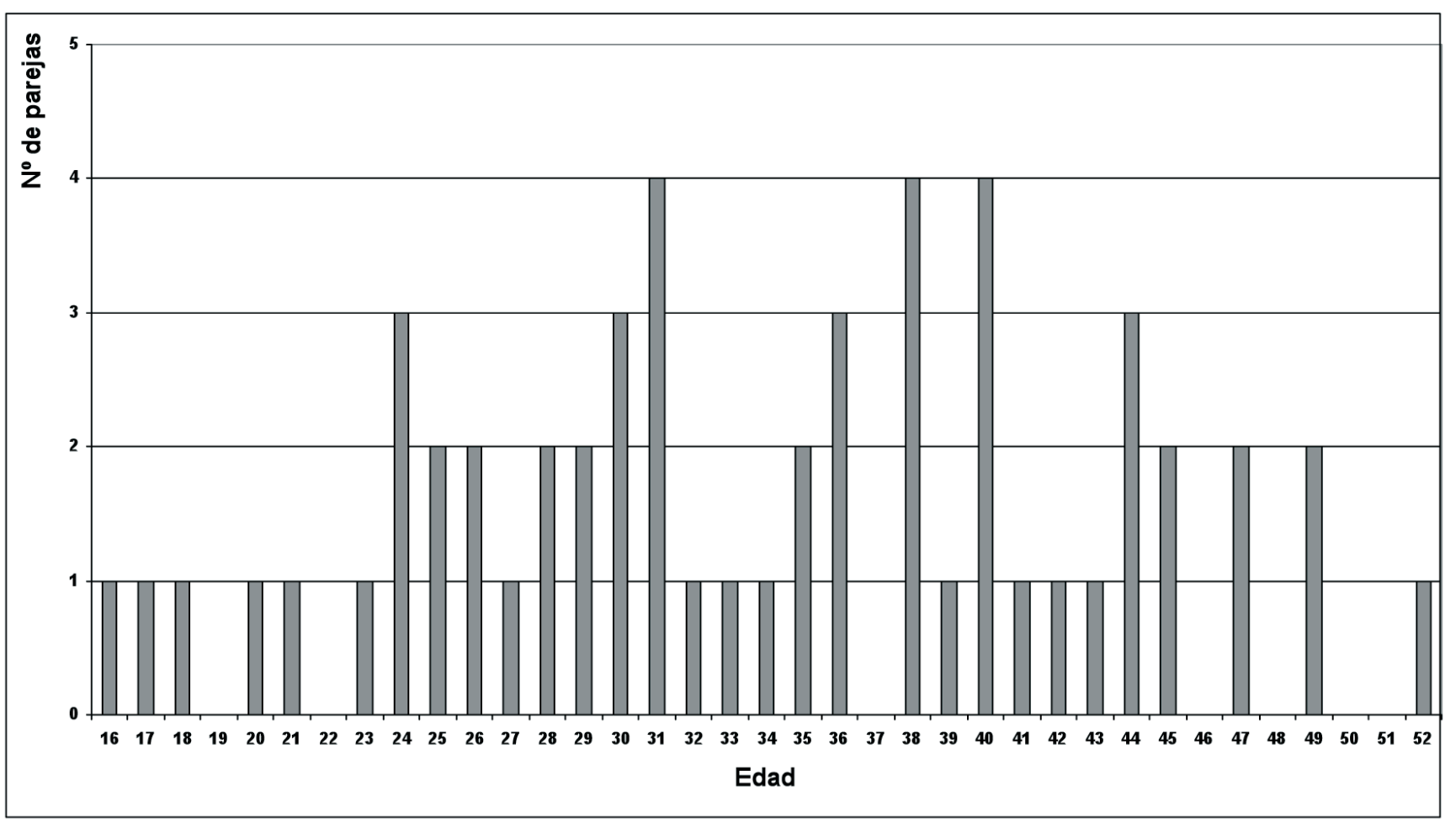

Figura 1. Distribución por edades de las parejas (caso clínico y control) que componen la muestra.

\section{Instrumentos}

Se administró el Inventario del Temperamento y el Carácter-Revisado (Temperament and Character Inventory-Revised; TCl-R; Cloninger, 1999), en la versión española de Fernández Aranda, Badía, Bayón y Aitken (2004). Consta de 240 ítems (5 de ellos de validez) que se contestan en una escala tipo Likert de 5 opciones. Ha sido validado en población general española (Gutiérrez-Zotes, Bayón, Montserrat, Valero, Labad, Cloninger et al., 2004) y estudiadas sus propiedades psicométricas en amplias muestras de estudiantes (Garabito, García-Valcárcel, Gutiérrez, Hernández, Luna, Pérez-Rodríguez et al., 2002) y de adictos a sus- tancias (Pedrero, 2006a), así como en otros idiomas, como el danés (Kristensen, 2006), el francés (Pelissolo, Mallet, Baleyte, Michel, Cloninger, Allilaire et al., 2005) o el italiano (Fossati, Cloninger, Villa, Borronia, Graziolia, Giarollia et al., 2007).

Las 7 dimensiones del modelo que evalúa el TCl-R son: Búsqueda de Novedad (tendencia heredada a responder fuertemente a estímulos nuevos y señales de que conduce a una actividad exploratoria en búsqueda de recompensas así como en la evitación de la monotonía y castigo), Evitación del Daño (tendencia a responder de forma intensa a las señales y estímulos aversivos, por lo que se aprende a inhibir la conduc- 
ta con el fin de evitar el castigo, la incertidumbre y la frustración), Dependencia de Recompensa (tiene que ver con el refuerzo social e implica variaciones neurobiológicas en cuanto a la sensibilidad a las señales sociales, el malestar en relación con la separación social y dependencia de la aprobación por otros) y Persistencia (mantenimiento de la conducta a pesar de refuerzos intermitentes) como rasgos temperamentales, y Autodirección (habilidad de la persona para controlar, regular y adaptar la conducta ajustándola a la situación de acuerdo con sus propias metas y valores), Cooperatividad (comportamientos éticos o prosociales, capacidad de aceptar, identificarse y colaborar con los demás) y Autotrascendencia (agrupa características de espiritualidad, misticismo, pensamiento mágico y religioso, así como la visión de uno mismo como parte integral del universo) como rasgos caracteriales.

\section{Procedimiento}

Los participantes fueron extraídos de la base de datos del CAD 4 San Blas (Instituto de Adicciones. Ayuntamiento de Madrid). La muestra de adictos forma parte de otra mayor, obtenida mediante muestreo consecutivo, de los sujetos que inician tratamiento por abuso/dependencia de sustancias. La muestra de población general se obtuvo mediante la técnica de bola de nieve (Goodman, 1961) como parte de otra mayor, utilizada en estudios de validación. Como criterio de inclusión se buscaron parejas de una y otra muestra que presentaran el mismo sexo, la misma edad y el mismo nivel de estudios. Se obtuvieron así 55 parejas equiparadas.

\section{Análisis de datos}

Se utilizó el paquete estadístico SPSS 12.0 para Windows. En todos las comparaciones de medias se utilizó la prueba $U$ de Mann-Whitney, dado el reducido número de integrantes en cada grupo y la falta de homogeneidad de varianzas en algunas de las dimensiones.

\section{RESULTADOS}

\section{Diferencias por grupos}

En la Tabla 1 puede apreciarse que las diferencias entre el grupo de adictos y el de población general se centraron en tres dimensiones. La Búsqueda de Novedad fue significativamente más alta entre los adictos, mientras que tanto la Persistencia como, y muy especialmente, la Autodirección, fueron significativamente más bajos que en la población general. En el caso de la primera, las diferencias afectaron a 3 de las cuatro subdimensiones (Impulsividad, Extravagan- cia y Desorden), mientras que en el caso de la Autodirección fueron 4 de las 5 subdimensiones las que se diferenciaron significativamente (Responsabilidad, Orientación, Recursos y Congruencia) y sólo dos de las cuatro subdimensiones en el caso de la Persistencia (Trabajo y Perfeccionismo). No se apreció significación en las diferencias en el resto de dimensiones ni subdimensiones.

\section{Diferencias por sexo}

Los varones adictos puntuaron significativamente más alto que los de población general en la escala de Búsqueda de Novedad, algo que también sucedió en el caso de las mujeres. Las adictas, además, puntuaron significativamente más bajo que las de población general en Autodirección, algo que no sucedió en el caso de los varones por sólo 6 milésimas. No apareció significación en las diferencias observadas en el resto de escalas del TCl-R (ver Tabla 2).

En cuanto a las subdimensiones, tanto varones como mujeres adictos puntuaron más que los de población general en Impulsividad ( $p<0,001$ y $p<0,05$, respectivamente) y Extravagancia ( $p<0,001$ y $p<0,01$, respectivamente) y menos en Congruencia $(p<0,01$ y $p<0,001$, respectivamente). Los varones adictos, además, puntuaron por debajo de los de población general en Perfeccionismo $(p<0,05)$, mientras que las mujeres adictas puntuaron menos que las de población general en Trabajo, Responsabilidad, Orientación, Recursos y Altruismo ( $p<0,05$ en todos los casos).

\section{Diferencias por edad}

En el grupo de menores de 20 años no apareció significación en las diferencias en ninguna de las dimensiones del TCl-R entre adictos y población general ( $N=3$ para cada grupo). Entre los que tienen entre 20 y 30 años apareció significación $(Z=-3,1 ; p<0,01)$ en Búsqueda de Novedad, que fue superior en los adictos (media $=64,9 ;$ D.T. $=6,5$ ) que en la población general (media $=51,2 ;$ D.T. $=13,0)(\mathrm{N}=15$ para cada grupo). Entre los que tienen entre 30 y 40 años ( $N=20$ en cada grupo), se mantuvieron las diferencias en Búsqueda de Novedad (adictos media=62,1; D.T.=10,6; población general media $=53,5 ;$ D.T. $=11,4 ; p<0,05)$, pero aparecieron también en Persistencia (adictos media $=44,5$; D.T.=10,0; población general media $=53,3 ;$ D.T. $=8,7$; $p<0,01$ ) y en Autodirección (adictos media $=35,3$; D.T.=13,5; población general media $=47,5$; D.T. $=10,0$; $p<0,01)$. Finalmente, en el grupo de los mayores de 40 años ( $N=17$ en cada grupo) se mantuvieron las diferencias en Búsqueda de Novedad (adictos media $=63,6$; D.T. $=9,4$; población general media $=49,1$; D.T. $=8,4$; $\mathrm{p}<0,001$ ) y en Autodirección (adictos media $=36,6$; D.T.=10,5; población general media $=43,8$; D.T. $=8,4$; $p<0,05)$ y aparecieron también en Dependencia de Recompensa (adictos media $=55,2 ;$ D.T. $=11,0$; población general media $=46,4 ;$ D.T. $=5,7 ; p<0,01)$. En la 
Tabla 1. Diferencias entre medias y su significación en las escalas y subescalas delTCI-R obtenidas por el grupo de adictos en tratamiento $(\mathrm{N}=55)$ y el grupo de población general emparejado

( $N=55)$ (Prueba U de Mann-Whitney); puntuaciones tipificadas, $m=50$, D.T.=10, según las puntuaciones normativas ofrecidas por Gutiérrez Zotes et al., 2004

\begin{tabular}{|c|c|c|c|c|c|c|}
\hline \multirow[b]{2}{*}{ TCl-R } & \multicolumn{2}{|c|}{ POBLACIÓN GENERAL } & \multicolumn{2}{|c|}{ ADICTOS } & \multirow[b]{2}{*}{ Z } & \multirow[b]{2}{*}{ Sig. } \\
\hline & Media & D.T. & Media & D.T. & & \\
\hline BÚSQUEDA DE NOVEDAD & 51,9 & 10,8 & 62,9 & 9,2 & $-5,1$ & $p<0,0001$ \\
\hline Excitabilidad Exploratoria & 51,7 & 8,7 & 54,6 & 9,0 & $-1,7$ & 0.09 \\
\hline Impulsividad & 49,1 & 9,8 & 59,3 & 11,0 & $-4,5$ & $p<0,0001$ \\
\hline Extravagancia & 51,9 & 12,4 & 63,9 & 9,6 & $-4,9$ & $p<0,0001$ \\
\hline Desorden & 52,6 & 11,3 & 57,5 & 9,9 & $-2,2$ & $p<0,05$ \\
\hline EVITACIÓN DEL DAÑO & 53,0 & 11,3 & 54,2 & 12,5 & $-0,5$ & 0,64 \\
\hline Preocupación & 54,3 & 10,1 & 57,7 & 12,0 & $-1,6$ & 0.11 \\
\hline Miedo a lo incierto & 50,0 & 9,8 & 48,5 & 12,0 & $-0,7$ & 0.48 \\
\hline Timidez & 51,7 & 10,3 & 50,8 & 11,7 & $-0,7$ & 0.47 \\
\hline Fatigabilidad & 52,2 & 12,7 & 54,0 & 12,9 & $-0,4$ & 0.68 \\
\hline DEPENDENCIA DE RECOMPENSA & 49,5 & 8,4 & 51,0 & 13,2 & $-1,3$ & 0.20 \\
\hline Sentimentalismo & 50,4 & 10,9 & 53,4 & 11,8 & $-1,7$ & 0.08 \\
\hline Calidez & 49,7 & 9,2 & 50,5 & 13,5 & $-0,9$ & 0.38 \\
\hline Apego Comunicatividad & 48,4 & 10,5 & 50,2 & 11,9 & $-1,2$ & 0.23 \\
\hline Dependencia & 50,0 & 10,2 & 48,7 & 10,1 & $-0,7$ & 0.48 \\
\hline PERSISTENCIA & 50,1 & 10,2 & 47,1 & 10,9 & $-2,1$ & $p<0,05$ \\
\hline Esfuerzo & 46,9 & 11,0 & 48,8 & 10,1 & $-0,5$ & 0,65 \\
\hline Trabajo & 48,9 & 9,8 & 43,5 & 11,9 & $-2,7$ & $p<0,01$ \\
\hline Ambición & 52,7 & 9,8 & 51,2 & 10,3 & $-1,1$ & 0,29 \\
\hline Perfeccionismo & 50,9 & 11,0 & 45,8 & 10,1 & $-2,6$ & $p<0,01$ \\
\hline AUTODIRECCIÓN & 43,7 & 11,9 & 36,0 & 11,8 & $-3,3$ & $p<0,001$ \\
\hline Responsabilidad & 44,5 & 13,0 & 37,1 & 14,2 & $-2,8$ & $p<0,01$ \\
\hline Orientación & 46,1 & 12,4 & 40,0 & 15,5 & $-2,2$ & $p<0,05$ \\
\hline Recursos & 47,8 & 10,4 & 42,5 & 12,7 & $-2,4$ & $p<0,05$ \\
\hline Autoaceptación & 45,0 & 10,3 & 44,5 & 10,0 & $-0,3$ & 0,78 \\
\hline Congruencia & 45,5 & 11,2 & 35,5 & 11,4 & $-4,4$ & $p<0,0001$ \\
\hline COOPERATIVIDAD & 46,8 & 11,1 & 45,4 & 11,5 & $-0,8$ & 0,80 \\
\hline Tolerancia social & 46,2 & 12,0 & 48,0 & 11,6 & $-0,8$ & 0.44 \\
\hline Empatía & 49,7 & 8,4 & 47,2 & 12,6 & $-0,8$ & 0.42 \\
\hline Altruismo & 48,7 & 9,0 & 44,4 & 10,6 & $-1,8$ & 0.07 \\
\hline Compasión & 46,6 & 12,8 & 47,3 & 12,6 & $-0,2$ & 0.83 \\
\hline Integridad & 48,5 & 9,6 & 47,1 & 9,7 & $-0,9$ & 0.36 \\
\hline AUTOTRASCENDENCIA & 51,9 & 11,0 & 55,3 & 11,6 & $-1,7$ & 0.09 \\
\hline Ensimismamiento & 51,7 & 11,5 & 56,2 & 11,6 & $-1,9$ & 0.05 \\
\hline Transpersonalidad & 50,7 & 9,3 & 51,6 & 12,4 & $-0,3$ & 0.73 \\
\hline Espiritualidad & 52,1 & 12,1 & 54,6 & 11,2 & $-1,4$ & 0.19 \\
\hline
\end{tabular}


Tabla 2. Diferencias, y su significación, por sexo entre adictos en tratamiento y población general, en las dimensiones delTCI-R (Prueba U de Mann-Whitney; puntuaciones tipificadas, m=50, D.T.=10, según las puntuaciones normativas ofrecidas por Gutiérrez Zotes et al., 2004.

\begin{tabular}{|c|c|c|c|c|c|c|c|c|c|c|c|c|}
\hline & \multicolumn{4}{|c|}{ VARONES } & \multicolumn{8}{|c|}{ MUJERES } \\
\hline & \multicolumn{2}{|c|}{$\begin{array}{l}\text { POBLACIÓN } \\
\text { GENERAL }\end{array}$} & \multicolumn{2}{|c|}{ ADICTOS } & \multirow[b]{2}{*}{ Z } & \multirow[b]{2}{*}{ Sig. } & \multicolumn{2}{|c|}{$\begin{array}{l}\text { POBLACIÓN } \\
\text { GENERAL }\end{array}$} & \multicolumn{2}{|c|}{ ADICTAS } & \multirow[b]{2}{*}{$\mathbf{Z}$} & \multirow[b]{2}{*}{ Sig. } \\
\hline & Media & D.T. & Media & D.T. & & & Media & D.T. & Media & D.T. & & \\
\hline BÚSQUEDA DE NOVEDAD & 50,9 & 10,8 & 62,2 & 9,7 & $-4,2$ & 0,000 & 53,7 & 10,8 & 64,1 & 8,4 & $-3,0$ & 0,003 \\
\hline EVITACIÓN DEL DAÑO & 52,0 & 9,9 & 52,0 & 12,0 & $-0,1$ & 0,939 & 54,8 & 13,5 & 58,0 & 12,8 & $-0,6$ & 0,570 \\
\hline $\begin{array}{l}\text { DEPENDENCIA DE } \\
\text { RECOMPENSA }\end{array}$ & 48,9 & 8,5 & 52,9 & 11,5 & $-1,8$ & 0,080 & 50,6 & 8,2 & 47,8 & 15,5 & $-0,2$ & 0,860 \\
\hline PERSISTENCIA & 49,9 & 10,3 & 47,3 & 11,3 & $-1,5$ & 0,132 & 50,5 & 10,3 & 46,7 & 10,5 & $-1,4$ & 0,151 \\
\hline AUTODIRECCIÓN & 43,4 & 10,5 & 38,5 & 11,6 & $-1,9$ & 0,056 & 44,2 & 14,2 & 31,5 & 10,9 & $-2,9$ & 0,004 \\
\hline COOPERATIVO & 47,0 & 9,6 & 47,2 & 9,7 & $-0,5$ & 0,613 & 46,6 & 13,7 & 42,4 & 13,8 & $-1,0$ & 0,310 \\
\hline AUTOTRASCENDENCIA & 52,8 & 11,1 & 56,3 & 10,1 & $-1,3$ & 0,188 & 50,2 & 10,9 & 53,6 & 14,0 & $-0,8$ & 0,401 \\
\hline
\end{tabular}

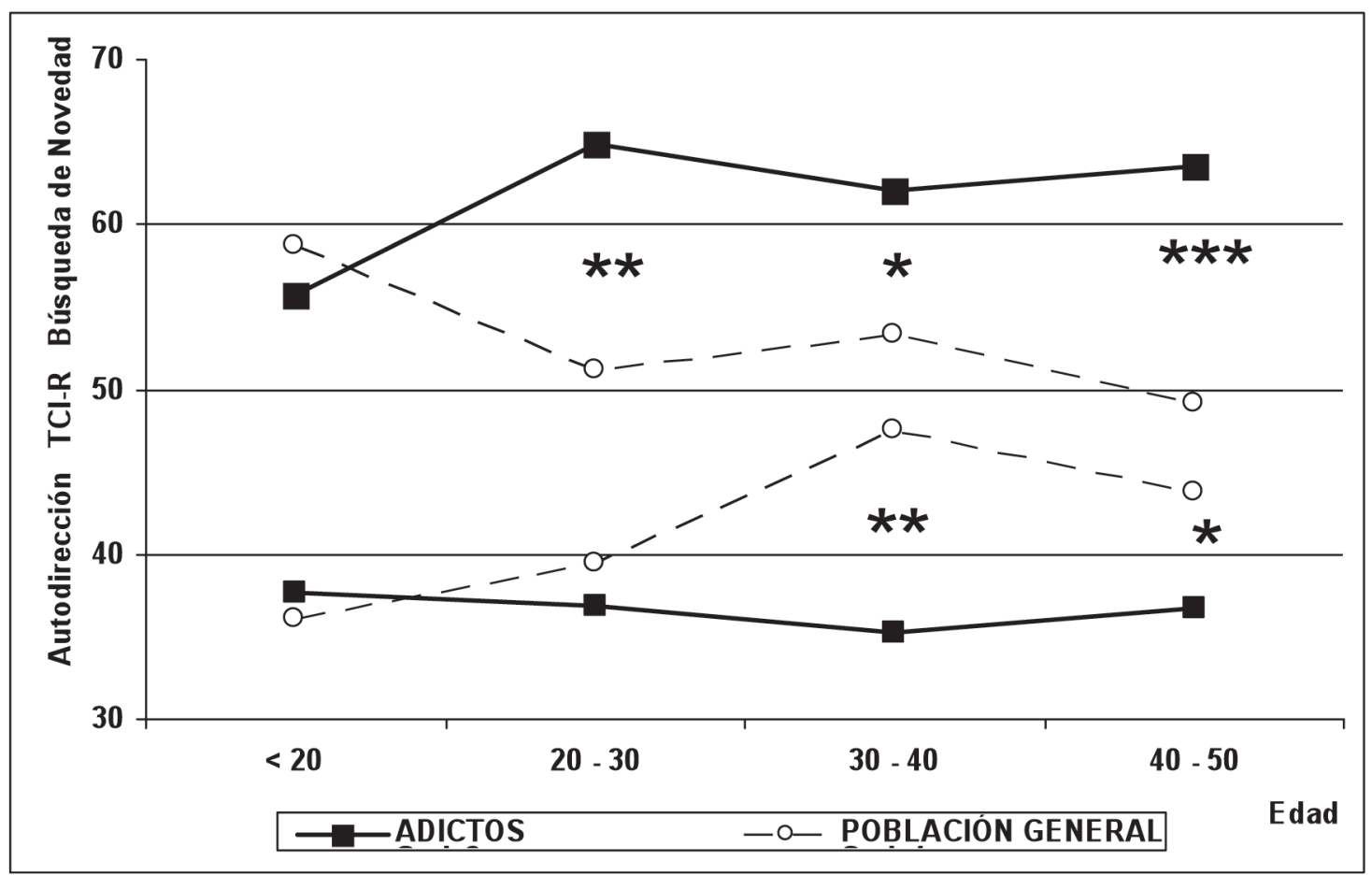

Figura 2. Se sumarizan las diferencias encontradas entre adictos y población general en las dimensiones críticas (Búsqueda de Novedad y Autodirección) según tramos de edad.

\section{Diferencias según nivel de estudios}

Las únicas diferencias significativas entre quienes sólo tienen completados estudios primarios se observaron en la dimensión de Búsqueda de Novedad, siendo los sujetos adictos quienes obtuvieron mayores puntuaciones (adictos media $=61,0 ;$ D.T. $=8,4$; población general media $=50,1 ;$ D.T. $=10,5 ; p<0,05$ ). Lo mismo sucedió entre quienes finalizaron estudios secundarios (adictos media $=63,8$; D.T. $=10,7$; pobla- ción general media $=54,5 ; D . T .=10,0 ; p<0,05)$. En los universitarios, las diferencias, además de a la Búsqueda de Novedad (adictos media $=63,1 ;$ D.T. $=8,8$; población general media $=51,0 ;$ D.T. $=11,4 ; p<0,001$ ), afectaron a la Autodirección (adictos media $=32,7$; D.T. $=10,6$; población general media $=45,4$; D.T. $=10,1$; $p<0,001$ ) y a la Persistencia (adictos media $=44,2$; D.T. $=11,3$; población general media $=50,7$; D.T. $=11,7$; $p<0,01)$. 


\section{DISCUSIÓN}

Los datos obtenidos en el presente estudio muestran que son dos las dimensiones que ofrecen diferencias netamente significativas a través de los diversos análisis: la Búsqueda de Novedad y la Autodirección. Estos resultados limitan las diferencias encontradas en otros estudios que no controlan las variables sexo, edad y nivel de estudios (Pedrero, 2006a), permitiendo considerar que son estos rasgos los que marcan una distinción real entre adictos y no adictos.

La Búsqueda de Novedad está relacionada, en la formulación teórica de Cloninger, con el sistema dopaminérgico. La investigación ha proporcionado un apoyo parcial a esta relación teórica (Schinka, Letsch y Crawford, 2002). Por otra parte, la implicación del sistema dopaminérgico es crucial en el desarrollo de la adicción, desde las primeras fases en que el consumo de droga empieza como una conducta instrumental dirigida a un objetivo, hasta la consolidación de la adicción como hábito compulsivo, controlado por mecanismos estímulo-respuesta, que invade, progresivamente, todas las esferas de la vida del individuo (Corominas, Roncero, Bruguera y Casas, 2007). La Impulsividad es el constructo que puede vincular a los dos anteriores; de hecho, la impulsividad es una de las subdimensiones del rasgo de Búsqueda de Novedad en la teoría de Cloninger y su instrumento de medida, el TCl-R. Y es la Impulsividad, junto con la Extravagancia, las que presentan diferencias más significativas en el presente estudio, entre adictos y población general. Sin embargo, la Impulsividad se ha estudiado tanto en relación al sistema serotoninérgico (Carver y Miller, 2006) como al dopaminérgico (Smillie y Jackson, 2005), por lo que no parece clara la relación entre Impulsividad y Búsqueda de Novedad.

De hecho, la dimensión de Búsqueda de Novedad en el TCl-R parece englobar al menos dos constructos hasta cierto punto independientes. La subdimensión de Excitabilidad Exploratoria no parece medir un constructo relacionado con la Impulsividad: presenta baja consistencia interna, pobre correlación con la escala total y disminuye sensiblemente la consistencia de ésta. Además, su patrón de correlaciones con escalas de trastornos de la personalidad es sensiblemente diferente al del resto de subdimensiones de Búsqueda de Novedad (Pedrero, 2006a), algo que también sucede cuando se exploran sus correlaciones con otras variables, como estrategias de afrontamiento (Pedrero, 2007a). En apariencia, y aunque serían necesarios estudios para elucidarlo, la Excitabilidad Exploratoria podría relacionarse con lo que Dickman (1990) denominó Impulsividad Funcional, mientras que la subescala de Impulsividad lo haría con la modalidad Disfuncional. La Impulsividad Funcional sería una disposición, es decir, un rasgo de personalidad, que se relacionaría con la capacidad de tomar decisiones rápi- das cuando la situación lo requiere, con el objetivo de obtener ganancias. La Impulsividad Disfuncional, por el contrario, consistiría en una disposición relacionada con la toma de decisiones rápidas e irreflexivas, que suelen tener consecuencias negativas para el individuo $y$, sin embargo, se repiten una y otra vez. Y es en esta última donde se aprecian diferencias significativas entre adictos y población general.

Cabe pensar si tal Impulsividad Disfuncional se asocia a los trastornos adictivos como predisponentes o como consecuentes. Es decir, si personas con predisposición a actuar irreflexivamente, sin atender a las consecuencias, son más proclives a establecer vínculos anómalos con las sustancias o si son éstas las que, por repetición, acaban modificando los sustratos neuronales y, en consecuencia, la conducta, conduciendo a la repetición irreflexiva del hábito adictivo. La segunda opción parece fuera de toda duda y se han propuesto teorías que vinculan la conducta adictiva a la sensibilización de las vías neuronales afectadas por el consumo (Robinson y Berridge, 2003). Además, parece que esta sensibilización, y su expresión conductual en modalidades impulsivas, está relacionada con la exposición temprana a la sustancia (Dom, Hulstijn y Sabbe, 2006).

El hecho de que en la mayor parte de las muestras la Búsqueda de Novedad aparezca más puntuada entre los adictos que entre los no adictos (p.e., Wills, Vaccaro y McNamara, 1994) no supone que éste sea un rasgo previo: un estudio exploró la capacidad explicativa de la hipótesis de la automedicación (el rasgo disfuncional predispone a la adicción) frente al modelo de la desviación (los sujetos involucrados en conductas desviadas modifican la expresión de sus rasgos en aras de una readaptación), no encontrando apoyo empírico para la primera y sí para la segunda (Chakroun, Doron y Swendsen, 2004). Otra posibilidad, de interés creciente en la investigación, es que sea la sustancia la que modifique los sustratos neuronales de la conducta. La impulsividad se comprende actualmente como un fallo de la inhibición, siendo ésta una de las facetas de la disfunción ejecutiva prefrontal (Kalenscher, Omán y Güntürkün, 2006). Contamos con evidencias suficientes sobre los efectos de diversas drogas sobre la función ejecutiva prefrontal: heroína (Pau, Lee y Chan, 2002), cocaína (Jovanovski, Erb, y Zakzanis, 2005), éxtasis (Montgomery, Fisk, Newcombe y Mur, 2005), alcohol (George, Rogers y Duka, 2005) y tabaco (Dinn, Aycicegi y Harris, 2004). En nuestro estudio, y como puede observarse en la Figura 2, la Búsqueda de Novedad es incluso superior en los no adictos menores de 20 años (aunque este dato carece de relevancia ante lo reducido de los grupos) y es entre los 20 y 30 años cuando este rasgo alcanza puntuaciones máximas en los adictos y las mayores diferencias con los de población general. Aunque el $\mathrm{N}$ de la muestra es insuficiente en todos 
los grupos, cabe considerar que el incremento en las puntuaciones de este rasgo, y especialmente en Impulsividad, sea efecto de la administración repetida de la sustancia. Los estudios que proponen una preferencia selectiva por una u otra sustancia en función de las diferencias temperamentales (Conway, Kane, Ball, Poling y Rounsaville, 2003; Le Bon, Basiaux, Streel, Tecco, Hanak, Hansenne et al., 2004) deberían revisarse ante la hipótesis alternativa de que tales diferencias lo que en realidad reflejaran es la acción selectiva de la sustancia sobre diversas estructuras corticales (p.e., la cocaína sobre los circuitos orbitofrontales, la heroína sobre los dorsolaterales y el alcohol sobre los medial-frontales).

En cuanto a la posibilidad de que determinados rasgos favorezcan el desarrollo de hábitos y conductas adictivas, el acuerdo no es general. Algunos estudios longitudinales encuentran muy poca o nula asociación entre rasgos de personalidad, medidos en la adolescencia, y la instauración del hábito de fumar a diario (Chassin, Presson, Pitts y Sherman, 2000; White, Pandina y Chen, 2002), mientras que otros encuentran que rasgos como alto Neuroticismo y alta Extraversión están significativa e independientemente asociados a una mayor probabilidad de ser fumadores en la edad adulta, si bien con efectos muy pequeños (Munafo y Black, 2007) y que únicamente se relacionan con un contacto temprano con la droga, pero no necesariamente con el desarrollo de una conducta adictiva (Audrain-McGovern, Rodriguez, Tercyak, Cuevas, Rodgers y Patterson, 2004). No obstante, una revisión reciente (Verdejo, Lawrence y Clark, 2008) parece encontrar más apoyo empírico al planteamiento según el cual la impulsividad es un antecedente del consumo.

El otro rasgo afectado por diferencias significativas entre adictos y población general en prácticamente todas las comparaciones es la Autodirección. Este rasgo mide disposiciones a organizar la conducta y dirigirla a metas, la asunción de responsabilidades derivadas de las propias decisiones, la adecuada gestión de los recursos disponibles y un sentido general de coherencia entre la propia concepción del sí mismo, las metas establecidas y las estrategias utilizadas para alcanzarlas. Como puede observarse en la Figura 2, los menores de 20 años de ambos grupos presentan puntuaciones críticamente bajas, como corresponde a su nivel de maduración. Sin embargo, desde los 20 años y hasta los 40, la capacidad de dirigir la propia vida crece en los sujetos de población general, hasta alcanzar los niveles promedio observados en la muestra normativa, declinando levemente poco después. En cambio, en los sujetos adictos esta capacidad apenas se desarrolla con el paso del tiempo, manteniéndose en los mismos niveles críticamente bajos que en la etapa adolescente. Y ello independientemente del sexo y del nivel de estudios, exhibiendo nuestra muestra de adictos universitarios una puntuación media dos desviaciones típicas por debajo de la muestra normativa, por sólo media de la muestra de universitarios no adictos. Lo que estos datos sugieren es que el contacto con la droga bloquea, de algún modo, la capacidad de establecer las propias metas y dirigir la conducta hacia ellas.

Pero sucede que esta capacidad de dirigir, planificar y ejecutar la conducta programada también requiere de la función ejecutiva cerebral, siendo éstos sus principales cometidos. De modo que el contacto temprano con las sustancias y, principalmente, la repetición del consumo, pueden afectar también al proceso de aprendizaje madurativo por dos vías: a partir de la modificación de los sustratos neuronales de la planificación/ejecución y a partir de los efectos psicosociales del uso de determinadas sustancias. Por una parte, hemos mencionado estudios que muestran las alteraciones en las funciones cerebrales encargadas de la conducta planificada; por otro, existe evidencia empírica de que los rasgos de personalidad interaccionan con las condiciones ambientales, de modo que condiciones favorables benefician el desarrollo de un adecuado autocontrol y condiciones desfavorables actúan en sentido inverso y el uso de sustancias adquiriría funciones autorregulatorias (Wills y Dishion, 2004).

La importancia de estas consideraciones afecta a otras áreas de intervención en el tratamiento de los trastornos adictivos. Medicamentos de amplio uso en este ámbito provocan severo empeoramiento de la función ejecutiva (Kockelmann, Elger, y Helmstaedter, 2004; Lutz y Helmstaedter, 2005) y su uso puede estar bloqueando cualquier logro psicoterapéutico.

En conclusión, los datos del presente estudio sugieren que si controlamos variables como el sexo, la edad o el nivel de estudios, las únicas diferencias significativas entre adictos y no adictos se circunscriben a dos rasgos: la Búsqueda de Novedad y la Autodirección. Estas diferencias podrían no ser previas, sino consecutivas al uso repetido de las sustancias, lo que reflejaría más el efecto de las drogas sobre los sustratos neuropsicológicos de la conducta que predisposiciones a la adicción. Son necesarios más estudios para confirmar estas hipótesis, aunque existen algunas evidencias que ofrecen un sólido sustento. De confirmarse estas hipótesis las implicaciones de cara a la clínica serían de considerable interés. Los programas de tratamiento deberían incorporar, además del estudio de la personalidad como patrón global de comportamiento estable, una evaluación y un abordaje de rehabilitación neuropsicológicos, ya disponible para lesiones similares de diferente etiología (Muñoz y Tirapu, 2001) y las tradicionales modalidades de intervención psicoterapéutica en trastornos adictivos (p.e., entrevista motivacional, prevención de recaí- 
das, trabajo con redes sociales) deberían reenfocarse hacia los sustratos neurales implicados (Lorea, Tirapu, Landa y López-Goñi, 2005), sin cuya recuperación el proceso de rehabilitación del trastorno adictivo puede tornarse estéril. El desafío es interdisciplinar: el área psicoterapéutica debería incorporar conceptos neuropsicológicos, el área médica debería considerar el uso de fármacos que pudieran obstaculizar el proceso de rehabilitación y otras áreas, hasta ahora secundarias, como la terapia ocupacional, deberían tomar un protagonismo creciente ante la incorporación de técnicas de rehabilitación neurocognitiva.

La limitación de este estudio es que se trata de un estudio transversal con una muestra pequeña de sujetos de ambas poblaciones. A pesar de que el control de las variables covariantes permite aquilatar las diferencias más significativas sería necesario contrastar los resultados en muestras más amplias o utilizando métodos de control estadístico que permitieran el control de las variables sin reducción del N, como puede ser un modelo multivariante. Se ha prestado atención sólo a las diferencias obtenidas con una alta significación $(p<0,001)$ para evitar la comisión del Error tipo I. Posiblemente, el uso de métodos estadísticos multivariantes encontrara diferencias más amplias, con más significación y con un tamaño del efecto suficiente como para ampliar las conclusiones del presente estudio.

\section{REFERENCIAS}

Audrain-McGovern, J., Rodriguez, D., Tercyak, K. P., Cuevas, J., Rodgers, K. y Patterson, F. (2004). Identifying and characterizing adolescent smoking trajectories. Cancer Epidemiology, Biomarkers, and Prevention, 13, 2023-2034

Barrón, E, Mateos, M. y Martínez-Villate, I. (2004). Temperamento y carácter en adictos a cocaína y a opiáceos. Adicciones, 16, 287-294.

Berrocal, C., Ortiz, M., Fierro, A. y Jiménez, J. A. (2001). Variables clínicas y de personalidad en adictos a heroína. Anuario de Psicología, 32, 67-87.

Brooner, R. K., Schmidt, C. W. Jr. y Herbst, J. H. (2002). Personality trait characteristics of opioid abusers with and without comorbid personality disorders. En P.T.COSTA Jr. y A.WIDIGER (Eds.): Personality disorders and the Five-Factor model of personality $\left(2^{2}\right.$ Ed.): Washington: American Psychological Association.

Carver, C. S. y Miller, C. J. (2006). Relations of serotonin function to personality: current views and a key methodological issue. Psychiatry Research, 30, 1-15.

Cervera, G., Haro, G., Martínez-Raga, J., Bolinches, F., de Vicente, P. y Valderrama, J. C. (2001). Los trastornos relacionados con el uso de sustancias desde la pers- pectiva de la psicopatología y las neurociencias. Trastornos Adictivos, 3, 164-171.

Chakroun, N., Doron, J. y Swendsen, J. (2004). Substance use, affective problems and personality traits: test of two association models. Encephale, 30, 564-569.

Chassin, L., Presson, C. C., Pitts, S. C. y Sherman, S. J. (2000). The natural history of cigarette smoking from adolescence to adulthood in a midwestern community sample: Multiple trajectories and their psychosocial correlates. Health Psychology, 19, 223-231.

Cloninger, C. R. (1999). Temperament and character inventory revised. Documento no publicado.

Conway, K. P., Kane, R. J., Ball, S. A., Poling, J. C. y Rounsaville, B. J. (2003). Personality, substance of choice, and polysubstance involvement among substance dependent patients. Drug \& Alcohol Dependence, 71, 65-75.

Corominas, M., Roncero, C., Bruguera, E. y Casas. M. (2007). Sistema dopaminérgico y adicciones. Revista de Neurología, 44, 23-31.

Dickman, S. J. (1990). Functional and dysfunctional Impulsivity: Personality and cognitive correlates. Journal of Personality and Social Psychology, 58, 95-102.

Dinn, W. M., Aycicegi, A. y Harris, C. L. (2004). Cigarette smoking in a student sample: neurocognitive and clinical correlates. Addictive Behaviors, 29, 107-126.

Dom, G., Hulstijn, W. y Sabbe, B. (2006). Differences in impulsivity and sensation seeking between earlyand late-onset alcoholics. Addictive Behaviors, 31, 298-308.

Esbaugh, D. M. (1982). Typologial analysis of MMPI personality pattern of drug dependence females. Journal of Personality Assesment, 46, 488-494.

Fernández-Aranda, F., Bayón, C., Badía, A. y Aitken, A. (2004). TCl-R, versión española. Manuscrito no publicado, 2004. Disponible en URL [consultado 12/08/2007]: https://psychobiology.wustl.edu/documents/TClRespana.pdf.

Fossati, A., Cloninger, C. R., Villa, D., Borronia, S., Graziolia, F., Giarollia, L. et al. (2007). Reliability and validity of the a version of the Temperament and Character InventoryRevised in an outpatient sample. Comprehensive Psychiatry, 48, 380-387.

Garabito, C., García-Valcárcel, B., Gutiérrez, E., Hernández, A., Luna M. A., Pérez-Rodríguez, M. M. et al. (2002). Validación del inventario del temperamento y el carácter (TCl-R). Modelo de personalidad de Cloninger. XV Congreso de Estudiantes de Medicina Preventiva y Salud Pública y Microbiología: Hábitos saludables en el S.XXI. Disponible en URL [consultado 19/07/2007]: http://www.uam.es/departamentos/medicina/preventiva/ especifica/congresoXV-30.html.

George, S., Rogers, R. D. y Duka, T. (2005). The acute effect of alcohol on decision making in social drinkers. Psychopharmacology, 182, 160-169.

Goodman, L. (1961). Snowball sampling. Annals of Mathematical Statistics, 32, 148. 
Gossop, M. R. y Eysenck, S. B. G. (1980). A further investigation into the personality of drug addicts in treatment. Addiction, 75, 305-311.

Gutiérrez-Zotes, J. A., Bayón, C., Montserrat, C., Valero, J., Labad, A., Cloninger, C. R. et al. (2004). Inventario del Temperamento y el Carácter-Revisado (TCl-R). Baremación y datos normativos en una muestra de población general. Actas Españolas de Psiquiatría, 32 8-15.

Gutiérrez-Zotes, J. A., Cortés, M. J., Valero, J., Peña, J. y Labad, A. (2005). Propiedades psicométricas de la versión española abreviada del TCl-R (TCl-140) y su relación con las Escalas de la Personalidad Psicopatológica (MMPI-2 PSY-5) en pacientes. Actas Españolas de Psiquiatría, 33, 231-237.

Janowsky, D. S., Hong, L., Morter, S. y Howe. L. (1999). Underlying personality differences between alcohol/ substance-use disorder patients with and without an affective disorder. Alcohol and Alcoholism, 34, 370-377.

Jovanovski, D., Erb, S. y Zakzanis, K. K.. (2005). Neurocognitive deficits in cocaine users: a quantitative review of the evidence. Journal of Clinical \& Experimental Neuropsychology, 27, 189-204.

Kalenscher, T., Ohmann, T., Güntürkün, O. (2006). The neuroscience of impulsive and self-controlled decisions. International Journal of Psychophysiology, 62, 203-211.

Kockelmann, E., Elger, C. E. y Helmstaedter, C. (2004). Cognitive profile of topiramate as compared with lamotrigine in epilepsy patients on antiepileptic drug polytherapy: relationships to blood serum levels and comedication. Epilepsy \& Behavior, 5, 716-721.

Le Bon, O., Basiaux, P., Streel, E., Tecco, J., Hanak, C., Hansenne, M., et al. (2004). Personality profile and drug of choice; a multivariate analysis using Cloninger's TCl on heroin addicts, alcoholics, and a random population group. Drug \& Alcohol Dependence, 73, 175-82.

Lutz, M. T. y Helmstaedter, C. (2005). EpiTrack: Tracking cognitive side effects of medication on attention and executive functions in patients with epilepsy. Epilepsy \& Behavior, 7, 708-714.

Marchiori, E., Loschi, S., Marconi, P. L., Mioni, D. y Pavan, L. (1999). Dependence, locus of control, parental bonding, and personality disorders: a study in alcoholics and controls. Alcohol \& Alcoholism, 34, 396-401.

McLellan, T. (1980): Development of psychiatric disorders in drug abusers: Relation between primary drug and type of disorder. Problems of Drug Dependence, NIDA Research Mongraph, 27, 149-155.

Montgomery, C., Fisk, J. E., Newcombe, P. y Mur, P. N. (2005). The differential effects of ecstasy/polydrug use on executive components: shifting, inhibition, updating and access to semantic memory. Psychopharmacology, 182, 262-276.

Munafo, M. R. y Black, S. (2007). Personality and smoking status: A longitudinal analysis. Nicotine \& Tobacco Research, 9, 397-404.
Muñoz. J. M y Tirapu, J. 82001). Rehabilitación neuropsicológica. Madrid: Síntesis.

Pau, C. W. H., Lee, T. M. C. y Chan, S. F. (2002). The impact of heroin on frontal executive functions. Archives of Clinical Neuropsychology, 17, 663-670.

Pedrero, E. J. (2002). Estudio de la personalidad de drogodependientes mediante el 'Big Five Questionnaire' (BFQ). Trastornos Adictivos, 4, 138-150.

Pedrero, E. J. (2003). Los trastornos de la personalidad en drogodependientes desde la perspectiva de los cinco grandes factores. Adicciones. 15, 203-220.

Pedrero, E. J. (2006a). Temperamento, carácter y trastornos de la personalidad. Aplicación del TCl-R a una muestra de consumidores de drogas en tratamiento y su relación con el MCMI-II. Adicciones, 18, 135-148.

Pedrero, E. J. (2006b). TCl-140: propiedades psicométricas, relación con el $\mathrm{TCl}-\mathrm{R}$ y con variables de personalidad. Estudio de una muestra de adictos en tratamiento. Trastornos Adictivos, 8, 155-167.

Pedrero, E. J. (2007a). Adaptación española de la Escala Multiaxial de Afrontamiento-Disposicional (Strategic Approach to Coping Scale-Dispositional Form; SACS-D) a población general y adictos a sustancias. Tesis no publicada. Madrid: UNED.

Pedrero, E .J. (2007b). El modelo de cinco factores y la estructura dimensional de los trastornos de la personalidad: estudio de una muestra de adictos a sustancias con el BFQ y el MCMI-II. Trastornos Adictivos, 9, 116-125.

Pelissolo, A., Mallet, L., Baleyte, J. M., Michel, G., Cloninger, C. R., Allilaire, J. F. y Jouvent, R. (2005). The Temperament and Character Inventory-Revised (TCl-R): psychometric characteristics of the French version. Acta Psychiatrica Scandinavica, 112, 126-133.

Robinson, T. E. y Berridge, K. C. (2003): Addiction. Annual Review of Psychology, 54, 25-53.

Sánchez-Hervás, E. y Berjano, E. (1996). Características de personalidad en sujetos drogodependientes. Psicothema, 8, 457-463.

Schinka, J. A., Letsch, E. A. y Crawford, F. C. (2002). DRD4 and novelty seeking: results of meta-analyses. American Journal of Medical Genetics, 114, 643-648.

Schuckit, M. A., Klein, J., Twitchell, G. y Smith, T. (1994) Personality test scores as predictors of alcoholism almost a decade later. American Journal of Psychiatry, $151,1038-1042$

Smillie, L. y Jackson, C. J. (2005). Functional Impulsivity and Reinforcement Sensitivity Theory. Journal of Personality, 74, 47-83.

Valenciano, L. (1936), Sobre la personalidad de los morfinistas. En: Archivos de Neurología en la Guerra Civil (1997). Archivos de Neurobiología, 67 (Supl. 1), 271-304

Verdejo, A., Lawrence, A. J., y Clark, L. (2008). Impulsivity as a vulnerability marker for substance use disorders: Review of findings from high-risk research, problem gamblers and genetic association studies. Neuroscience \& Biobehavioral Reviews, 32, 777-810. 
White, H. R., Pandina, R. J. y Chen, P. H. (2002). Developmental trajectories of cigarette use from early adolescence into young adulthood. Drug and Alcohol Dependence, 65, 167-178.

Wills, T. A. y Dishion, T. J. (2004). Temperament and adolescent substance use: a transactional analysis of emerging self-control. Journal of Clinical Child \& Adolescent Psychology, 33, 69-81.

Wills, T. A., Vaccaro, D. y McNamara, G. (1994). Novelty seeking, risk taking, and related constructs as predictors of adolescent substance use: an application of Cloninger's theory. Journal of Substance Abuse, 6, 1-20. 
